\title{
Research and Simulation of Ultra Wideband Based on Rake Receiver

\author{
Xuewen $\mathrm{He}^{1, \mathrm{a}}$, Yanmeng Wang ${ }^{1, \mathrm{~b}}$
} \\ ${ }^{1}$ School of Mechanical and Electrical Engineering Jiangxi University of Science and Technology
}

Ganzhou, 341000, China

ahxw993@vip.163.com , blongand1204@163.com

Keywords: Ultra-wideband (UWB); Multipath; Rake receiver; performance

\begin{abstract}
The Ultra Wideband signal with multiple multipath component and use the Rake receiver can combine energy of multipath components. Using Rake reception, the performance of three kinds of receiving methods is compared with binary Ultra Wideband signal using the Maximal Ratio Combining (MRC), and got the simulation results.
\end{abstract}

\section{Introduction}

Ultra-wideband (UWB) technology is a rapid development of the new communication mode in recent years. It has the space transmission capacity, high ability in distinguish ability of multipath , low power spectral density, good in signal disguised, high anti-jamming and anti-multipath capability[1], become a hot issues at home and abroad in communication field.

The bandwidth of Ultra-wideband pulse signal is more than $500 \mathrm{MHz}$ and the transmission bandwidth can reach $7.5 \mathrm{GHz}$. In order to meet the performances of the receiver design, the key problem is to effective collect and use of energy method under UWB channel model. In UWB communication system, the signal of carry information is very short duration (periodic) pulse signal, the duty ratio are very low, so the resulting multipath component can separable in time and the separated multipath components can be used to collect the emission signal energy completely. Rake reception is used to collect multipath and against multipath fading [2].

The structure of Rake receiver is complicated and the cost to realize is high. However, due to it can improve the performance of system receiving, And with the development of science and technology, the realization of the difficulty and cost is reduced, so it has been widely used in the third generation wireless communication system. According to the UWB system can separate multipath concepts, when two signal multipath time delays are longer than the width of spread spectrum code piece, we can consider the two signals are not related or the path can separable. Response in frequency domain, which is the signal transmission bandwidth, is longer than the signal coherent bandwidth [3]. Due to the UWB system is broadband transmission, all the channels sharing frequency resources, so the UWB system can use Rake reception technology.

\section{Rake receiver basic principle}

Rake receiver is refers to use the different arrival delay to separate the signal parts, use multiple correlator to compose more related branches. These branches can extract energy from the corresponding multipath signal, branches output through the appropriate weighted combination to get multipath diversity gain [4]. Rake receiver can use irrelevant separable path capture the signal energy effectively, in order to overcome the fading and improve system reliability. According to the IEEE 802.15.3a statistical channel model, in single user condition, the receiving signal can be expressed as[5]

$$
\mathrm{r}(\mathrm{t})=\mathrm{X} \sqrt{E_{T X}} \sum_{j} \sum_{n=1}^{N} \sum_{k=1}^{K(n)} \alpha_{n k} a_{j} p_{0}\left(t-j T_{s}-\varphi_{j}-\tau_{n k}\right)+\mathrm{n}(\mathrm{t}) .
$$


In type (1): X--log-normal distribution amplitudes gain of the channel; $\mathrm{E}_{T X}$--the emission energy of each pulse; $a_{j}$--the amplitude of the Jth transmitted pulse (for PPM, $a_{j}=1$ ); $T_{s}$--Average pulse repetition period; $\varphi_{j}$--the timing jitter of the Jth pulse; $\tau_{n k}$--the delay of the Kth path in Nth cluster.

For each implementation of the channel impulse response, channel coefficient contains energy are normalized, that is

$$
\sum_{n=1}^{N} \sum_{k=1}^{K(n)}\left|\alpha_{n k}\right|^{2}=1
$$

So type (1) can be expressed as

$$
\mathrm{r}(\mathrm{t})=\sqrt{E_{R X}} \sum_{j} \sum_{n=1}^{N} \sum_{k=1}^{K(n)} \alpha_{n k} a_{j} p_{0}\left(t-j T_{s}-\varphi_{j}-\tau_{n k}\right)+\mathrm{n}(\mathrm{t}) .
$$

in Type (3) $\mathrm{E}_{R X}$ is total receiving energy of a transmitted pulse

$\mathrm{E}_{R X}=\mathrm{X}^{2} \mathrm{E}_{T X}$.

And is different from Additive White Gaussian Noise (AWGN) channel, the $\mathrm{E}_{R X}$ is spread over a period of time and appeared in different multipath components.

In Ultra Wideband Impulse Radio (UWB-IR) system, the pulse duration is in nanosecond level. We can assume that all the multipath components are mutually overlapped and received wave are formed by independent components. So we can improve receiver judgment performance by combining the same emission pulse of several independent multipath components.

Rake receiver is composed by correlator, combiner and detector. Fig. 1 is a RAKE receiver with

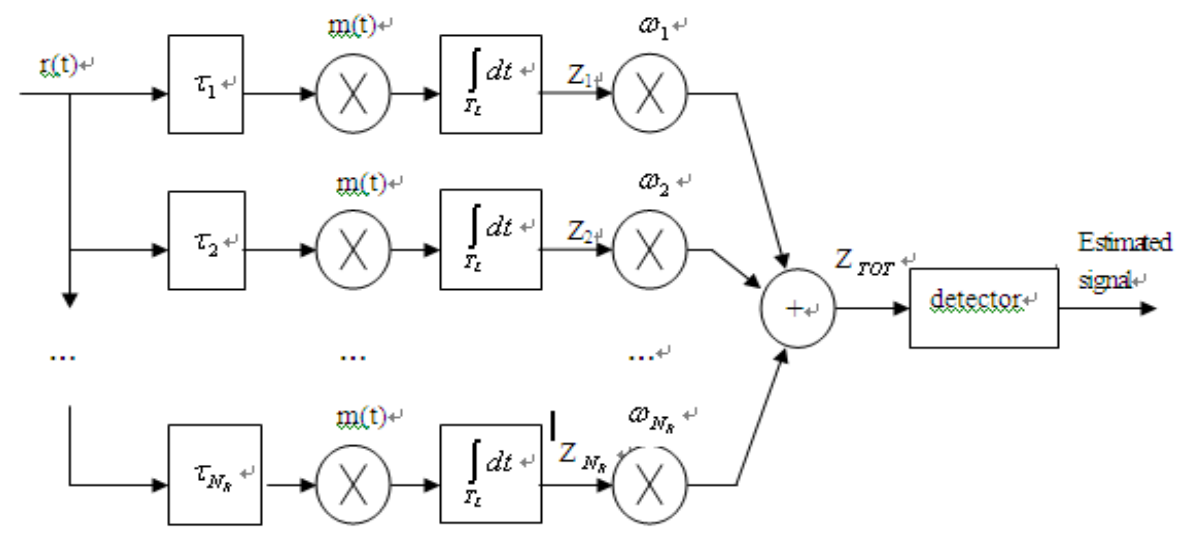

Fig.1 RAKE receiver with $\mathrm{N}_{R}$ parallel correlates and delay units

$\mathrm{N}_{R}$ parallel correlates and delay units; it contains many multiple correlates and matches with the

same transmitted pulse waveform of multiple multipath component phase, respectively. $T_{L}$ means

the duration of the channel impulse response, $\mathrm{Z}_{\mathrm{TOT}}$ is Rake combiner output decision variable and it was sent to detector, $\tau_{j}$ means the Jth path delay spread.

According to fig.1, all components can be aligned on time after time shift units and so it can makes all the branches using the same related mask $m(t)$. In this paper, the combiner choose maximal ratio combining (MRC).In MRC mode, different multipath components weighted first, and then merge together. The weighted factor is equal to the channel impulse response coefficient. $\mathrm{Z}_{\text {тот }}$ is an output decision variable of Rake combiner and is sent to detector, and then is used to transmit for judgment.

\section{Binary UWB signal Rake receiving performance}

UWB channel is a typical multipath channel[5], so signal energy is distribute in multiple multipath quantitatively when the pulse signal into the receiving end. Use Rake reception 
technology can combine multiple path of energy[6] and improve the receiving end output Signal-to-Noise Ratio.

Rake belongs to the reception [7].For binary PAM signal, assume that signal waveform $s_{1}(t)=g(t)$ and $\mathrm{s}_{2}(\mathrm{t})=-\mathrm{g}(\mathrm{t})$. In additive Gaussian channel, the receiving signal when send $\mathrm{s}_{1}(\mathrm{t})$ is

$\mathrm{r}(\mathrm{t})=\mathrm{s}_{1}(\mathrm{t})+\mathrm{n}(\mathrm{t})$

in type (5), $\mathrm{n}(\mathrm{t})$ indicates the additive Gaussian noise that the value is 0 , variance of N0/2, its bit error rate can be expressed as[7,8]

$$
\operatorname{Pr}_{e}=\mathrm{Q}\left(\sqrt{\frac{2 \varepsilon_{b}}{N_{0}}}\right) .
$$

Here $\mathrm{Q}(\alpha)=(2 \pi)^{-1 / 2} \int_{\alpha}^{\infty} \exp \left(-z^{2} / 2\right) d z$ is Complementary error function, $\varepsilon_{b}$ is the energy of waveform $g(t)$.

For binary PAM-UWB signals has similar situations, assuming that channel characteristics such as multipath amplitude and time delay are known, the literature [6] gives the receiving performance when use Rake reception, the bit error rate can be expressed as:

$$
\mathrm{P}_{\mathrm{r}_{e}}==\mathrm{Q}\left(\sqrt{\frac{2 E_{P} N_{P} \eta_{\text {capSR }}}{N_{0}}}\right) \text {. }
$$

in type(7), $N_{P}$ pulse parameters meaning for 1bit, where

$$
\begin{aligned}
& \eta_{\text {capSR }}=\frac{\left[\int_{-\infty}^{\infty} r(t) r_{\text {temp }}(t) d t\right]}{\int_{-\infty}^{\infty} r^{2}(t) \int_{-\infty}^{\infty} r_{\text {temp }}^{2}(t) d t} . \\
& E_{P}=\int_{-\infty}^{\infty} r^{2}(t) d t .
\end{aligned}
$$

$r_{\text {temp }}(t)$ is local reference signal, $\eta_{\text {cap }}$ is Rake receiver energy capture efficiency, usually associated with the selected branch ( Finger ) number and so on if $\eta_{\text {capsR }}=1$, the formula (6) is equivalent to (7).

\section{Rake receiver performance comparisons}

The signal used for UWB Rake receiver can be expressed as:

$$
\mathrm{m}(\mathrm{t})=\sum_{i=1}^{N} c_{i} w\left(t-\tau_{i}\right) \text {. }
$$

Different $\mathrm{N}$ represent different Rake receiver. For ARake, requires an infinite number of Rake branches that requires infinite number of correlates, combining all of the distinguished multipath components. To achieve this purpose, the receiver needs to be obtained all the path loss $c_{i}$ and delay $\tau_{i}$ in real time, The related template signal $\mathrm{m}(\mathrm{t})$ constructed fully matched with the received signal. Studies have shown that in the typical modern office building, Rake receiver to capture $60 \%$ of the total energy of the received waveform, need about 50 different Rake branch[5]. In order to reduce the complexity of the receiver, can use different multipath selection, the first is called Selective Rake (SRake), it is obtained $N_{s}$ strongest multipath component selected from the $\mathrm{N}$ multipath components from the receiver input. Then the branch number of Rake receiver can be reduced, but the receiver still need to track all the multipath component in order to select[9].Another receiver known as Partial Rake ( PRake), it has no selection process, direct merger the $N_{p}$ path which first to reach the receiving end, because no multipath selection, so relatively simple, but the performance can not be optimal[10].In order to described the receiving method of the above three more image, the simulation is shown in Figure 2-4, the results of Figure 2 with the vector ARake consistent. In extreme NLOS environment, assumed that the distance 
between the transmitter and the receiver is $8 \mathrm{~m}$, the reference attenuation $\mathrm{A}_{0}=51 \mathrm{~dB}$, attenuation index $\gamma=3.5, \Lambda=0.0667 / \mathrm{ns}, \lambda=2.1 / \mathrm{ns}, \Gamma=24, \gamma=12, \sigma_{\xi}=3.3941, \sigma_{\zeta}=3.3941 \mathrm{~dB}, \sigma_{g}=3 \mathrm{~dB}$. According to IEEE 802.15.SG3a, statistical model to obtain a channel discrete impulse response samples as Figure 2 show.

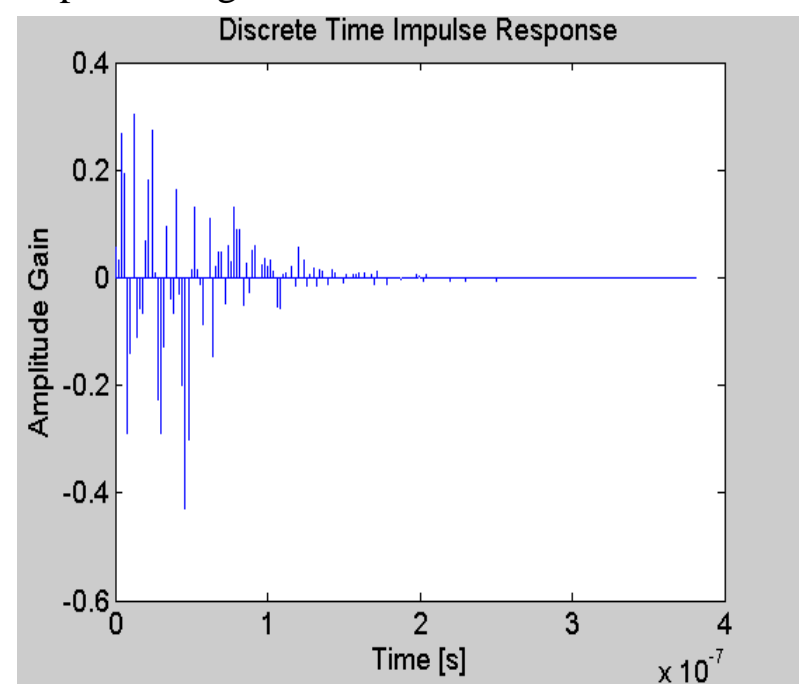

Fig.2 Extreme NLOS, discrete-time channel impulse response

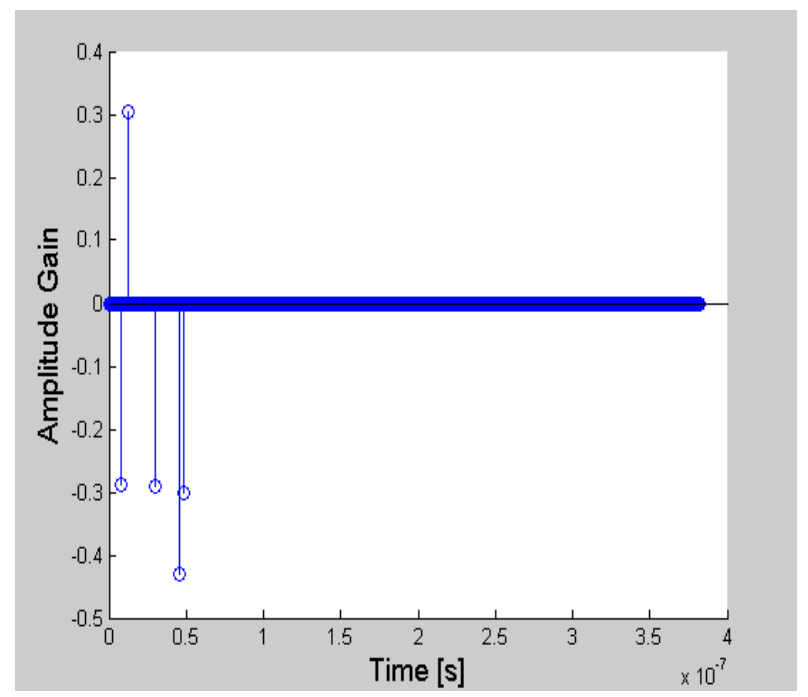

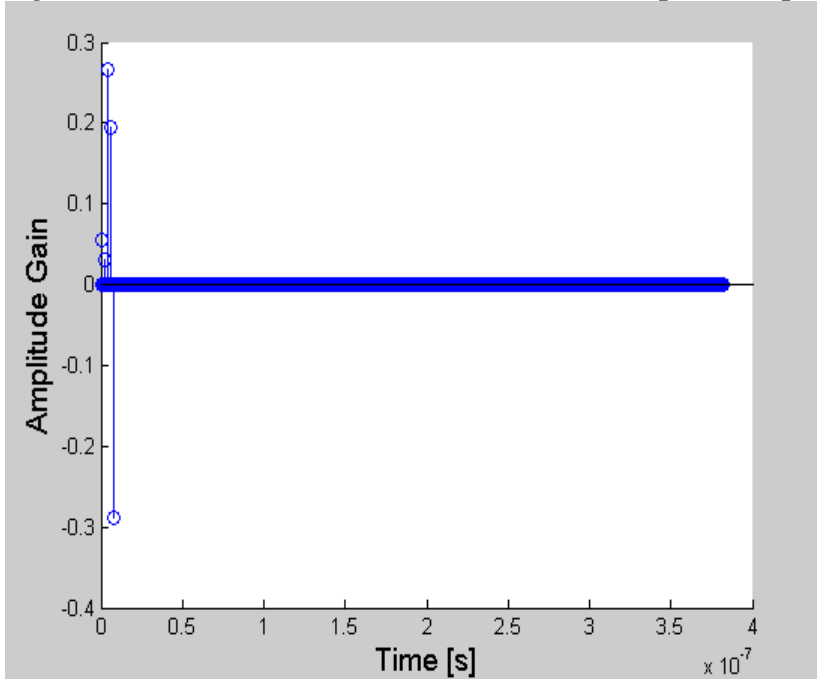

Fig.4 PRake channel valuation

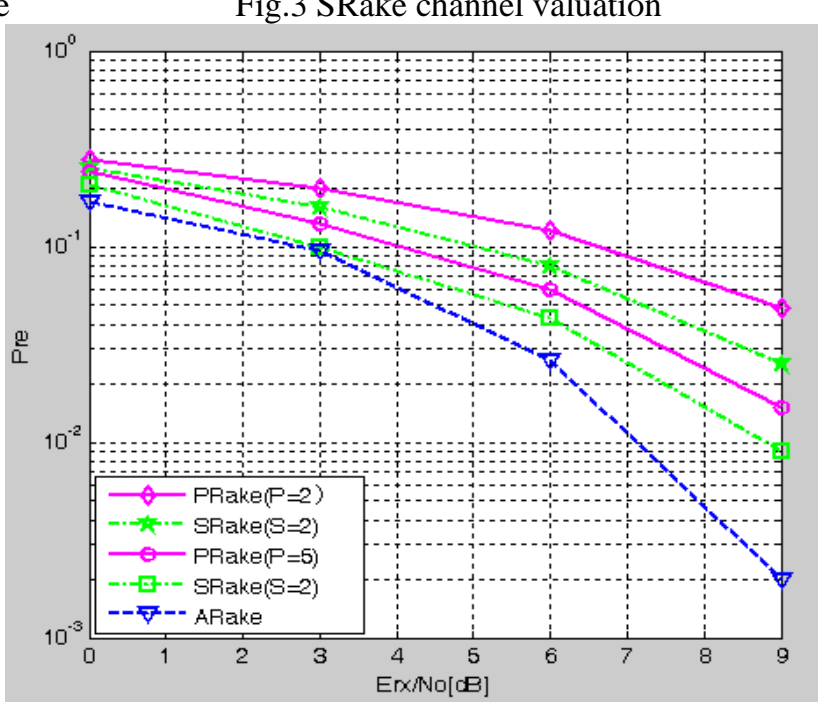

Fig.5 the Rake receiver bit error rate and Signal-to-Noise ratio curve

Using Rake receiver increases the complexity of the receiver, the more number of multipaths that analyzed and combined before judgment, the more complexity receiver has. Therefore, it is possible to reduce the receiver complexity by reducing the number of multipath components of the receiver processing. From the above it can be seen SRake and PRake receiver processing can reduce the number of multipath components, thereby reducing the complexity of the receiver. To analysis the bit error rate use two different approaches to reduce the Rake receiver complexity, and compare these two Rake receiving with the All Rake (ARake) receiving method which handle all the multipath components. When signals through the above channel, the Rake receiver performance curve that use three kinds of Rake receiving method shown in Figure 5.

Interpretation of result: ARake receiving performance was clearly better than SRake and PRake, but difficult to practical application. $\mathrm{P}$ and $\mathrm{S}$ represent the number of branches when using PRake and SRake receiving method respectively. The performance of the receiver is related to the number of branches no matter SRake or PRake, branch number, the more the better reception performance. And in the case of same number of branch, SRake receiver performance is superior to PRake receiver performance. 


\section{Summary}

Analysis of the three kinds of Rake receiver's working principle, from the five Rake receiver bit error rate and signal-to-noise ratio curve can see that ARake receiver system performance is best, but the design is too complex. When the number reaches a certain value, PRake performance and SRake is almost the same. In practice, PRake receiver structure is the most simple and most easy to achieve, but the bit error rate of SRake is batter than PRake. In the application process, considering the relationship between performance and complexity, and choosing the right Rake receiver.

\section{Acknowledgment}

Project supported by The National Natural Science Foundation of China (Grant NO. 61163063); Project supported by the Research Foundation of Education Bureau of Jiangxi Province, China (Grant No. GJJ12329).

\section{References}

[1]Lin Zhu, Xiao Yuan, Lijia Ge, et al. Ultra Wideband Wireless Sensor Network Summary [J].Measurement and control technology, 2004, 23(12):1-4.In Chinese

[2]Malik W Q, Stevens C J, Edwards D J. MultiPath Effects in Ultrawideband Rake Reception[J].IEEE Trans. On Antennas and Propagation, 2008, 56(2):507-514.

[3]Bartosz Mielczarek, M.O.Wessman, Arne Svensson. Performance of coherent UWB Rake Receivers with channel estimators[C].IEEE Vehicular Technology conference, 2004, 3(58):1880-1884.

[4]PRICE R, GREEN Jr P E. A communication technique for multipath channels [J]. Proc, IRE, 1958,46(3):55-570.

[5]Lijia Ge, Fanxin Zeng, Yulin Liu, et al. Ultra-Wideband Wireless Communication [M]. National Defence Industry Press, Beijing, 2005.8. In Chinese

[6]Wu L, Wu X, Tian Z. Asymptotically Optimal UWB Receivers With Noisy Templates: Design and Comparison With RAKE[J]. IEEE Journal on Selected Areas in Communications. 2006, 24(4): 808-814

[7]Scholtz R A, Pozar D, Won N. Ultra-Wideband Radio[J]. EURASIP Journal on Applied Signal Processing, 2005, 3:252-272

[8]Proakis J G. Digital Communications [M]. Lijun Zhang, et al translate. Publishing House of Electronics Industry, Beijing, 2003.1. In Chinese

[9]Jia Lou. RAKE receiver performance simulateion based on the IEEE UWB standard channel model [J].Journal of Chonqing University of posts and telecommunications, 2008.6:20-23. In Chinese

[10]Zhiqing Wan, Wancheng Ge. Research and Simulation of multipath Ultra-Wideband receiver [J]. Information Technology, 2010, (1): 26-33. In Chinese 